\title{
Performance Improvisation of Bituminous Roads using waste Plastic Material
}

\author{
Xianglin a, * \\ ${ }^{a}$ Department of Building Engineering, Tongji University, China.
}

*Corresponding Author profxia.lin@tongji.edu.cn (Xianglin)

Received : 10 04 2019 Accepted : 18 05 2019

\begin{abstract}
Utilization of waste plastic as a aggregate in bituminous mix results in weak bonding between the plastic and bitumen. This study reports on the feasibility of using plastic waste and chemical additives to improvise the performance and mechanical properties of bituminous mixtures. The selected recycled waste plastics were used as partial aggregate replacement in bituminous mix product. Strong oxidizing mixture of dichromate and sulphuric acid was used to treat the plastic, while the bitumen was treated with a cross linking agent, polyethylene mine. Three modified bituminous mixtures were prepared and the stiffness results were compared with the control bituminous mixture. It was observed that the stiffness increased by $10 \%$ for the chemically modified bituminous mixtures. This improvement is attributed to an increase in the bonding forces between the aggregates and the bitumen. Besides, a mechanism is proposed in order to explain the effect of the chemical additives on the increase in the stiffness of the bituminous mixture.
\end{abstract}

Keywords: Waste Plastic, aggregates, bituminous mixture, chemical additives, polyethyleneimine

\section{Introduction}

The properties of the constituent materials in bituminous pavements have an important influence on the life of the road pavement. Adherence or bonding is vital to the performance of a pavement. There is a lack of understanding of the fundamental chemistry and physics of these paving material components owing to the past reliance on tests which primarily use physical properties of bitumen and bitumen mixes that cannot be derived from a fundamental engineering analysis of their behaviour as a means of classification. Due to growth in various types of industries together with population growth has resulted in enormous increase in production of various types of waste materials. With the increasing usage of plastics in various sectors, the discharge of polymeric wastes is bound to create further greater environmental problems in the years to come. Attempts are still being made by various organizations and researchers to find methods for effective utilization of some of these waste materials. Of these, the efforts to find useful applications of some of the waste products in highway construction have shown encouraging results [1].

Replacing the primary aggregates partially with recycled waste plastic has the benefits of reducing the extraction of aggregates, the amounts of disposed waste plastics, and the associated environmental and social impacts. According to Waste Resources Action Program 2.8 million tons of waste plastics is generated per annum in the UK [2]. Similarly, out of the 0.5 million tons of the tyre waste created every year, only 0.06 million tons is actually recycled for use in roads [2]. While, the natural aggregates are supplied in vast quantities for a few pounds per ton; the processed recycled rubber and plastic can have a value of up to hundreds of pounds per ton. It is not economical to use these materials as alternative aggregates unless their use adds sufficient value to the bituminous product, so that the cost of the materials can be justified. Bitumen has been widely used in the construction of flexible pavements for a long time. In some applications, however, the performance of conventional bitumen may not be considered satisfactory. The bonding between bitumen and aggregate is of utmost importance as it is the primary characteristic that influences the integrity of the pavement. This bonding must be established at the initial stages of contact between the bitumen and the aggregate and must endure during the lifetime of the pavement. A large number of investigations [3 7] have demonstrated that bitumen properties (e.g., viscoelasticity and temperature suscep tibility) can be improved using an additive or a chemical reaction modification. These results have been further verified by various authors including [8 10]. Among various types of additives, chemical anti stripping agents are probably the most promising as reported by $[11,12]$. They list five primary mechanisms that either act individually or

Together to cause the de-bonding of bitumen from aggregate. These mechanisms are: 
1) Detachment, which is the separation caused by water of strongly influenced by the bitumen; meaning that under the bitumen film from the aggregate without any visual break in the bitumen film.

2) (2) Displacement, which results from the intrusion of water to the aggregate surface through a break in the bitumen film or through the film itself.

3) (3) Spontaneous emulsification, which is the formation of an inverted reversible emulsion at the aggregate.

4) (4) Pore pressure, which is the increased pressure caused by circulation of trapped water through the void structure of the aggregate.

5) (5) Hydraulic scouring, which occurs on surface courses because of a compression tension cycle caused by the interaction of tire pressure with surface water.

One of the methods to minimize the effect of water on the pavement is the use of liquid anti stripping agents are frequently used. As discussed earlier, the use of recycled waste plastics as partial aggregate replacement material in bituminous mix product suffers from weak bonding with the bitumen. Various authors including [13,14] have proposed plastic treatments by a physical or chemical method. Alternatively, Hirose et al. [15], The main objective of the study documented in this paper is to compare the engineering properties of a bituminous mixture containing treated plastic and treated bitumen with those containing untreated plastic and untreated bitumen. The samples are further compared with the standard bituminous mixtures.

\section{Materials and Methods}

The section has been divided into further sub sections to provide the rationale behind choosing and designing of the bituminous mixtures used for the Repeat Load Axial Test (RLAT) and the Repeat Load Indirect Tensile Test (RLITT).

\subsection{Choice of Bituminous Mixture Type for Testing}

As discussed earlier, the overall aim of this study is to examine the feasibility of modified bituminous mixtures by adding recycled waste plastic as a partial replacement of aggregate. Hence, it is essential to choose the right bituminous mixture that would display most clearly the benefits of the modification. Bituminous mixtures can be divided into two categories: (i) Hot Rolled Asphalt (HRA) and (ii) Bituminous Macadam. Usually the term "Asphalt" is used in context of its European nomenclature, referring to the particular type of mixtures of bitumen and aggregate. However, in North America it simply means bitumen. Hot Rolled Asphalt (HRA) mixes rely almost entirely on the bitumen and filler content of the mixture for their strength. The material relies heavily on the competence of the sand/bitumen fraction, and the mechanical properties are severe conditions, the HRA wearing course may rut. Bitumen is responsible for the viscoelastic behaviour characteristics of HRA and plays a dominant role in determining the resistance of HRA to rutting as reported by [16]. Hence, while the HRA mixtures may demonstrate changes in bitumen properties very well, but would be useless for demonstrating the effect of differing aggregate properties.

Unlike HRA, Bituminous macadam relies on the interlocking of the aggregate particles as the major contributor for its strength. Coated macadam, is a road material consisting of graded aggregate that has been coated with bitumen and for which the intimate interlocking of the aggregate particles is a major factor on the strength of the compacted material. Evidence provided by various authors [17 20], suggests that bitumen macadam may be the most suitable material for demonstrating the influence of replacing a part of aggregate by the polymer particles, on the bituminous mixtures. The $6 \mathrm{~mm}$ medium graded surface course is one kind of macadam surface layer, and has been selected in this work as the most appropriate materials. This material is commonly used as a paving material in playground area, footway surface, patching and mini paver work application.

\subsection{Materials}

For reasons discussed above, bitumen type $\mathrm{C}$ from Nynas Company was used in this work. Table 1 gives a summary of the physical properties of the bitumen. The following two grades of limestone aggregates: (i) $6 \mathrm{~mm}$ Limestone and (ii) Limestone Dust were used in this study. This type of aggregate is the most

Table 1. Physical Properties of Bitumen Type C

\begin{tabular}{|c|c|}
\hline Properties & 125 \\
\hline $\begin{array}{c}\text { Penetration(100 gram, 5 seconds } \\
25 \mathrm{C}^{\circ}(1 / 10)^{\text {th }} \text { of mm) [36] }\end{array}$ & 42 \\
\hline $\begin{array}{c}\text { Softening Point (C }) \\
\text { (Ring \& Ball Method) }\end{array}$ \\
\hline
\end{tabular}

Commonly used for road pavement in the UK. The experimental program included sieve analysis and gradation test on both kind of aggregates in order that the aggregate mixes satisfy therequirement of the B.S. 598 specifications (1990) for midpoint gradation have been selected and shown in Table 2 and Fig. 1(a). The selected recycled waste plastics (supplied by European Metal Recovery Ltd.) were used as partial aggregate replacement in bituminous mix product. The plastic comprised a mixture of plastic and rubber, 1 4 $\mathrm{mm}$ in size with a specific gravity of 0.9 and was 

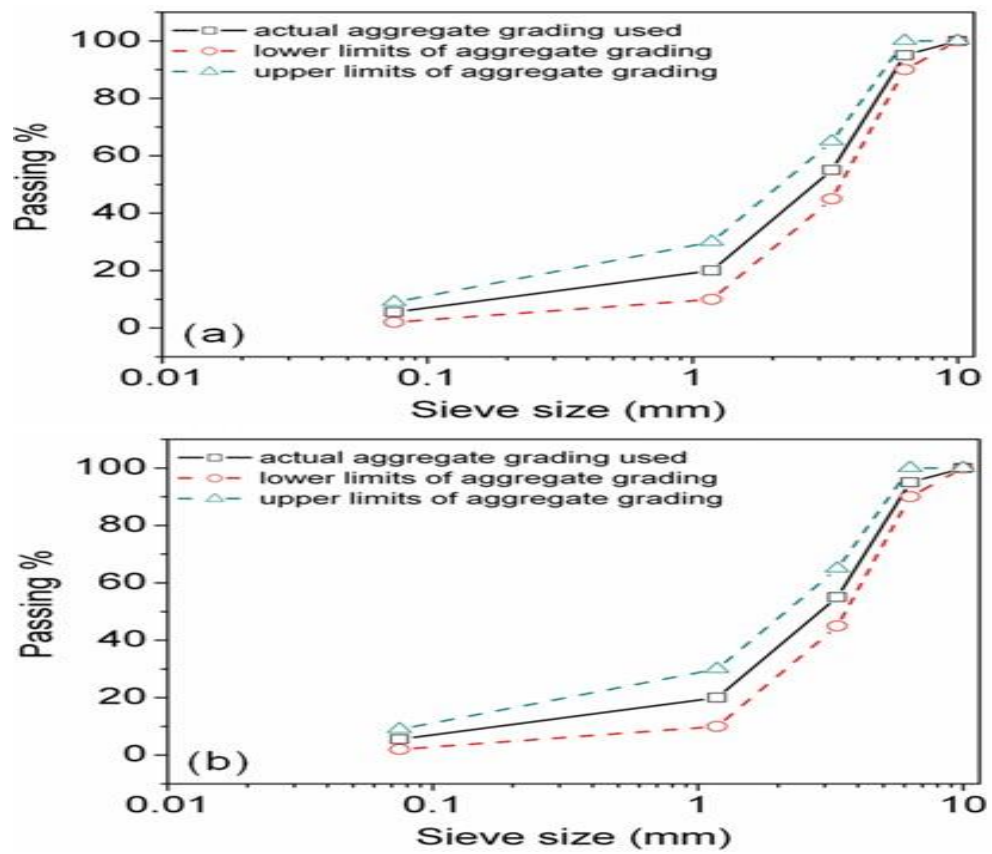

Fig. 1 (a) Gradation of Aggregates Adopted for 6 mm MGSC Mix, (b) Gradation of Aggregate and Plastic Adopted for $6 \mathrm{~mm}$ MGSC Mix
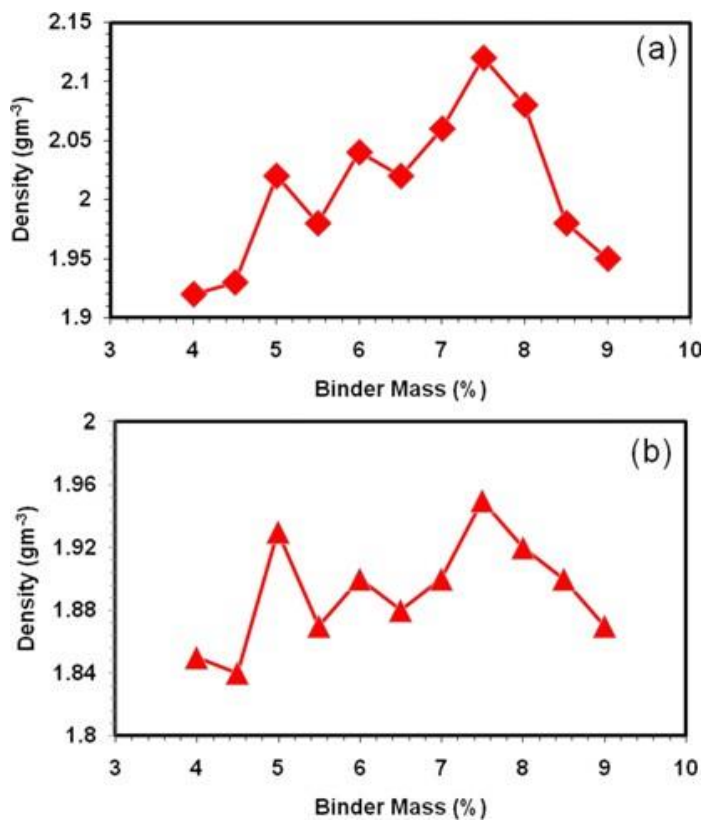

Fig 2. (a) Optimum Binder Content by Mix Density, (b) Optimum Binder Content by Compacted Aggregate Density

Recovered from scrapped cars. Grain size distributions of waste plastic in MGSC Mix are given in Table 3 and Fig. 1(b). The additive used to treat the waste plastic aggregate Particles were an oxidising solution of chromic acid, comprising of potassium dichromate, sulphuric acid and water in a ratio of $7: 12: 150$ by weight. The reaction time was 1 24 hours. Polyethylenimine 2 wt. \% (by bitumen) was used to treat the bitumen.

\subsection{Optimum Binder Content}

The optimum binder content for bitumen $C$ used to prepare a bituminous mixture in this work was $5.4 \%$. This is the case when only a mineral aggregate was used to prepare the product. It is necessary to find the optimum binder content when the recycled waste plastic was used as a partial replacement of the mineral aggregate. According to B.S.598 (1990) and B.S. 4987 part 1 (2001), at least nine binder contents of intervals at $0.5 \%$ of total mix with at least three binder contents on each side of the optimum value are recommended (4.0,4.5,5.0,5.5,6.0,6.5,7.0,7.5,8.0,8.5,9.0). The optimum binder content experiments were carried out for the following bituminous mixture:(Bitumen) $+8 \%$ EMR plastic. Four bituminous mixtures were prepared from each binder. From the mixture design plots (Figs. 2(a, b)), the optimum binder content for mix density were found to be 7.5\%. This optimum binder content was used with all bituminous mix products containing recycled waste plastics. 
Table 2. Aggregate Grading without Plastic \% Target Binder: 5.4

\begin{tabular}{|c|c|c|c|c|c|c|c|}
\hline Aggregate & $6 \mathrm{~mm}$ & Dust & & & & & Spec. \\
\hline$\%$ Blend & 50 & 50 & 100 & \multirow{2}{*}{ Mid Point } & $\%$ & & Limits \\
\hline Sieve $(\mathrm{mm})$ & & & Blend & & Variance $( \pm)$ & & \\
\hline 10 & 100 & 100 & 100.0 & 100 & 0.0 & 100 & 100 \\
\hline 6.3 & 96.1 & 90.9 & 93.5 & 95 & 1.5 & 90 & 100 \\
\hline 3.35 & 22.1 & 95 & 58.6 & 55 & 3.6 & 45 & 65 \\
\hline 1.18 & 3 & 46.3 & 24.7 & 20 & 4.7 & 10 & 30 \\
\hline 0.075 & 2.77 & 5.7 & 4.2 & 5.5 & 1.3 & 2 & 9 \\
\hline
\end{tabular}

Table 3. Aggregate Grading with Plastic \%Target Binder: 7.5

\begin{tabular}{|c|c|c|c|c|c|c|c|c|}
\hline Aggregate & $6 \mathrm{~mm}$ & Dust & Plastic & & & & \multirow{2}{*}{\multicolumn{2}{|c|}{ Spec. Limits }} \\
\hline$\%$ Blend & 46 & 46 & 8 & 100 & \multirow{2}{*}{ Mid Point } & $\%$ & & \\
\hline Sieve $(\mathrm{mm})$ & & & & Blend & & Variance $( \pm)$ & & \\
\hline 10 & 100 & 100 & 100 & 100.0 & 100 & 0.0 & 100 & 100 \\
\hline 6.3 & 96.1 & 90.9 & 100 & 94.0 & 95 & 1.0 & 90 & 100 \\
\hline 3.35 & 22.1 & 95 & 68.9 & 59.4 & 55 & 4.4 & 45 & 65 \\
\hline 1.18 & 3 & 46.3 & 4.8 & 23.1 & 20 & 3.1 & 10 & 30 \\
\hline 0.075 & 2.77 & 5.7 & 1.6 & 4.0 & 5.5 & 1.5 & 2 & 9 \\
\hline
\end{tabular}

Table 4. Bituminous Mixtures used in the Study

\begin{tabular}{|c|c|}
\hline Bituminous mixtures NO. & Specification \\
\hline $\begin{array}{c}\text { (Controlmix) } 1 \\
\sim(\mathrm{C})\end{array}$ & $\begin{array}{c}6 \mathrm{~mm} \text { medium graded surface course with 5.4\%Bitumen type c, o/6 (6 mm limestone and } \\
\text { limestone dust) }\end{array}$ \\
\hline $2 \sim(\mathrm{CP})$ & $\begin{array}{c}6 \mathrm{~mm} \text { medium graded surface course with } 7.5 \% \text { Bitumen type c, } 0 / 6(6 \mathrm{~mm} \\
\text { limestone and limestone dust) and } 8 \% \text { O } 4 \mathrm{~mm} \text { Waste PLASTIC by the weight of } \\
\text { total aggregate. }\end{array}$ \\
\hline $3 \sim(\mathrm{CTP})$ & $\begin{array}{c}6 \mathrm{~mm} \text { medium graded surface course with } 7.5 \% \text { Bitumen type c, } 0 / 6 \text { (6 } \mathrm{mm} \text { limestone } \\
\text { and limestone dust) with } 8 \% \text { O } 4 \mathrm{~mm} \text { TREATED waste Plastic by the weight of the totalb } \\
\text { aggregate. }\end{array}$ \\
\hline $4 \sim(\mathrm{TCTP})$ & $\begin{array}{l}\text { 6mmmediumgradedsurfacecoursewith } 7.5 \% \text { treatedBitumentypeco/6 }(6 \mathrm{mmlimestoneandlimes} \\
\text { toneDUST)AND } 8 \% \text { 0 } 4 \mathrm{~mm} \text { treated WASTE PLASTIC by the weight of total aggregate. }\end{array}$ \\
\hline
\end{tabular}

\subsection{Preparation of $6 \mathrm{~mm}$ Medium Graded Surface Course}

The $6 \mathrm{~mm}$ size Medium Graded Wearing Course specification chosen for the test was based on BS 4987: part 1 (2001). The proportions for the control mix and plastic are shown in Tables $1 \sim$ 3, respectively. Samples were made in $101.6 \mathrm{~mm}$ internal diameter Marshall Mould. Once the percentage met the specification, batch quantities were prepared for each sample. The samples were compacted using Marshall Testing Machine and were compacted for 50 blows each side. The specimens with plastic additives were immersed in cold water to control the swelling of the rubber and plastic. For this study the bituminous mixtures types were used as given in Table 4 . 


\subsection{Test Method}

\subsubsection{Repeat Load Indirect Tensile Stiffness}

A Lab VIEW ${ }^{\circledR}$ computer software was used to acquire the stiffness reading calculated from the applied loads and deformation recorded for each specimen. Five readings were taken for the pulsed loading in one axis. The mean of these five readings was recorded. A second axis was selected at an angle of $90^{\circ}$ to the first, and the test was repeated. The mean values of stiffness modulus from this second test were compared with the results of the first test. If the difference between the two results did not exceed $10 \%$, the mean of the two results was calculated and recorded as the stiffness for the specimen. However, if the difference between the two values was greater than that range above, then the results were rejected. Four samples from each type of the mixes were produced and the average stiffness results of the three samples were recorded.

\subsubsection{Sensitivity to Water}

Water sensitivity is the quality or state of a thin surfacing system, prepared so as to be suitable for traffic if it were part of a road pavement, following conditioning in water. Although many factors contribute to the de gradation of as phalt concrete pavements, however moisture remains a key element in the deterioration of the asphalt mixture. There are two mechanisms by which moisture can degrade the integrity of an asphalt concretematrix:

(i) Loss of cohesive strength and stiffness in the bitumen film that may be due to several mechanisms, and (ii) the failure of the adhesive bond between the aggregate and bitumen, often called stripping. Asphalt pavements which degrade in the presence of moisture are termed as water sensitive. Stripping is defined as the breaking of the adhesive bonds between the aggregate surface and the asphalt cement. When this bonding is broken, the pavement is weakened and various forms of water damage take place. All water sensitivity studies agree that stripping is caused by water.

Displacing the asphalt from the aggregate surface, but they vary in reporting stripping rates and in understanding the be havior at the asphalt aggregate interface in the presence of water. This emphasized the importance of understanding the mechanisms by which stripping occurs in order to adopt proper measures. The test procedure used involved the following steps:

1. The unconditioned stiffness designated as $\operatorname{ITSM}_{u}$ wasdetermined.

2. The specimen was placed in the vacuum desiccator,covered it with distilled water at $(20 \pm 1)^{\circ} \mathrm{C}$, the apparatus was sealed and a partial vacuum of $510 \pm 25 \mathrm{~mm} \mathrm{Hg}$ was applied for $(30 \pm 1$ minutes.
3. The specimen was removed from the vacuum desiccator.

4. The specimen was placed in a hot water bath at $60 \pm 1^{\circ} \mathrm{C}$ for $6 \pm 1$ hours. The sample was supported by a wire mesh basket to minimize distortion.

5. The specimen was removed from the hot water bath and placed in cold water bath at $5 \pm 1^{\circ} \mathrm{C}$ for 16 hours.

6. The specimen was removed from the cold water bath and immediately placed in a water ba that $20 \pm 0.5^{\circ} \mathrm{C}$ for atleast 2 hours.

7. The specimen was removed from water bath, surface dried and the conditioned stiffness was determined at a test tem premature of $(20 \pm 0.5)^{\circ} \mathrm{C}$ for the first conditioning cycle,desig natedasITSM $\mathrm{c}_{\mathrm{c} 1}$.

8. Steps 4 to 6 were repeated, the conditioned stiffness of the specimen for the second and up to tenth cycle was

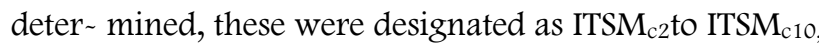
respectively.

The stiffness ratio (ITSMRatio,ci) for the specimens for each conditioning cycle was calculated asfollows:

ITSMRatio,ci= ITSMci/ ITSMu

Where

$\mathrm{Ci}=$ Conditioning cycle $\mathrm{i}=1$ to 10

$\mathrm{ITSM}_{\mathrm{ci}}=$ Conditioned stiffness after conditioning cycle ci

$\mathrm{ITSM}_{\mathrm{u}}=$ Unconditioned stiffness

\section{Results and Discussion}

The present investigation shows the finding of the laboratory investigations of plain and waste plastic modified dense bituminous macadam mixes. From the mixture design plots given in Figs. 1 and 2, the general trend observed is that the density of the mix increased with an increase in the binder content. The optimum binder content for mix density was determined to be $7.5 \%$ (by weight of aggregates). This optimum binder content was therefore used with all bituminous mix products containing the recycled waste plastics as the aggregate replacement material.

\subsection{Indirect Ten sile Strength}

From the indirect tensile strength test results, four types of the dense bituminous macadam mixes are shown in Table 5 and Fig. 3. From these results it is evident that the mixtures treated with the chemical additives exhibited improved stiffness compared with the untreated mixtures. When both the bitumen and the waste plastic were chemically treated, as in the mixture type 4 (TCTP), an improvement of $28 \%$ in the stiffness value was achieved.

The high stability and inert nature of plastic surface will weaken the attraction bonding force between the 
Table 5. Results of the Repeat Load Indirect Tensile Test

\begin{tabular}{|c|c|c|}
\hline Mixture Type & Stiffness (Mpa) & Density $\left(\mathrm{g} / \mathrm{cm}^{3}\right)$ \\
\hline C & 1876 & 2.4 \\
\hline CP & 1223 & 2.13 \\
\hline CTP & 1445 & 2.14 \\
\hline TCTP & 1688 & 2.16 \\
\hline
\end{tabular}

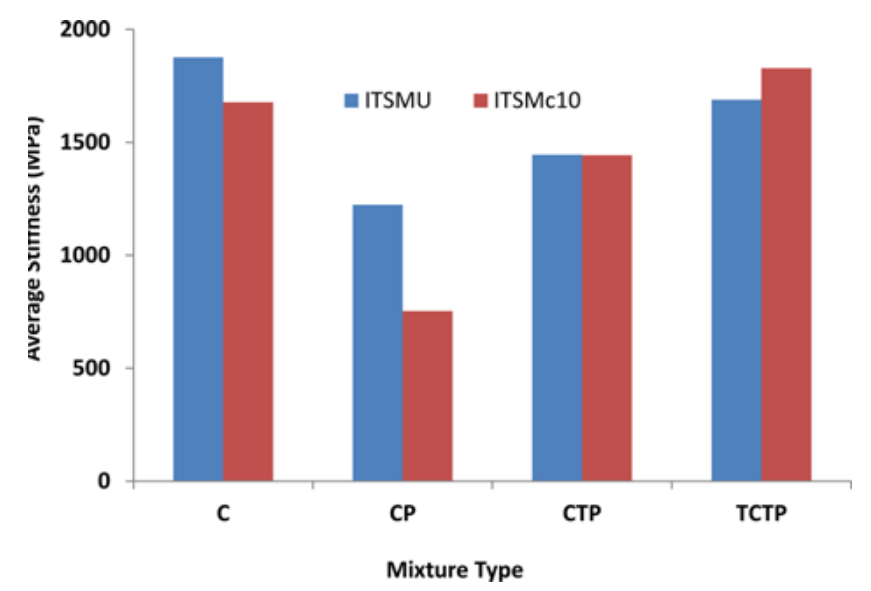

Fig 3. Unconditioned and Ten Cycle Conditioned Stiffness

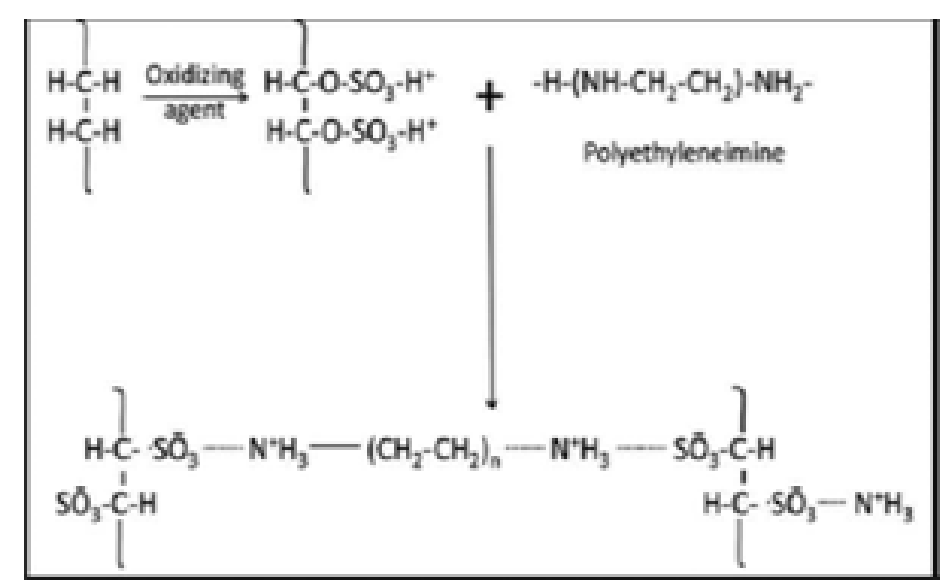

Fig. 4. Chemical Reaction for the Treatment of Bituminous Mixtures

Bitumen and the plastic surface. Hence the weak bond (weak adhesion) between the bitumen and the plastic particles appears to be the main reason for the observed reduction in the stiffness. The treatment of the plastic particles by strong oxidizing agent (dichromate/sulphuric acid solution) will activate the plastic surface through the oxidation process and will introduce active ionic functional groups on the plastic surfaces. These functional groups can then be reacted further in the presence of a crosslinking agent such as the polyethyleneimine in order to crosslink the plastic through hydrogen bonds with other bitumen constituents and hence enhancing bitumen/aggregate adhesion (See Fig. 4).

However, any benefit in terms of enhanced long term durability is a subject to further tests, for example, water sensitivity test.

\subsection{Water Sensitivity}

To investigate the mixtures sensitivity to water, the material was tested using the "retained stiffness after water immersion test” (ITSM Ratio) (BBA, 2000). 
Table 6. Summary of Sensitivity to Water Test

for Ten Cycle

\begin{tabular}{|c|c|c|c|c|c|c|c|c|c|c|c|}
\hline \multirow{2}{*}{$\begin{array}{l}\text { Mixture } \\
\text { Type }\end{array}$} & ITSMU & \multicolumn{10}{|c|}{$\begin{array}{c}\text { Stiffness Ratio } \\
\text { (ITSMU/ITSMC) }\end{array}$} \\
\cline { 3 - 12 } & & SR1 & SR2 & SR3 & SR4 & SR5 & SR6 & SR7 & SR8 & SR9 & SR10 \\
\hline $1 \sim(\mathrm{C})$ & 1876 & 1.1 & 1.1 & 1.00 & 1.00 & 1.10 & 0.97 & 1.00 & 1.00 & 1.00 & 0.89 \\
\hline $2 \sim(\mathrm{CP})$ & 1223 & 1.10 & 1.00 & 0.92 & 0.93 & 0.95 & 0.82 & 0.82 & 0.80 & 0.60 & 0.60 \\
\hline $\begin{array}{c}3 \sim \\
(\mathrm{CTP})\end{array}$ & 1445 & 1.00 & 1.00 & 1.00 & 1.10 & 1.00 & 1.00 & 1.17 & 1.10 & 1.00 & 1.00 \\
\hline $\begin{array}{c}4 \sim \\
\text { (TCTP) }\end{array}$ & 1688 & 1.20 & 1.10 & 1.10 & 1.15 & 1.2 & 1.16 & 1.18 & 1.2 & 1.2 & 1.1 \\
\hline
\end{tabular}

The results are summarized in Table 6. Of the four materials tested, the untreated plastic $\sim$ modified mixture (CP) exhibited an anomalous behaviour. Over the ten cycles, its stiffness was reduced by $40 \%$, whereas the chemically modified bituminous mixtures (TCTP) exhibited an increase in the stiffness by more than $10 \%$. This observed improvement is due to the increase in the bonding between the aggregates and the bitumen resulting from the action of the chemical additives Polyethylene mine (PEI). It is proposed that PEI can form complexes in the presence of oppositely charge monomers and can develop an interpenetrating network through cross linking of the polymer chains that comprise of primary polymer of the constituent bitumen. This complication leads to the strengthening and enhancement of the adhesive properties of the bitumen, which increases the bonding between the plastic surface and the bitumen and has been explained previously by, and Lu and Harvey [21].

\section{Conclusions}

The results of the study reveal that at optimal binder content $(7.5 \%$ by aggregate weight), the use of chemically treated plastic as a partial replacement, for aggregate in bituminous mixture provides a higher stiffness than untreated plastic. This is due to the enhancement of adhesion between the bitumen and the plastic surface particles. While it can be argued that the control samples involve less bitumen content; the mixture samples involve recycled waste plastic with high bitumen content. The use of chemically treated plastic together with chemically treated bitumen contributes more in increasing the strength of the bond between the aggregate and the bitumen. Over ten cycles of soaked conditions, the retained tensile strength of the treated plastic modified bituminous macadam mixes is found to be $10 \%$ higher than unconditioned sample. This high stability is due to the effect of the use of chemical additives which enhance the bonding of aggregates and the bitumen. For the economical utilization of waste plastic material, a further optimization of the process is necessary keeping in consideration the perceived benefits vs. the added cost. However, the results provide a better understanding of the chemistry and highlight the use of plastic waste in construction application which has two fold effects (i) it utilizes the waste which will otherwise go to landfills, which is inherently a high cost activity and increases the pollution and (ii) the improvement in the strength of the bitumen mixtures provides value added feature.

\section{References}

[1] R. L. Schroeder, The use of recycled materials in highway construction, Public Roads, 58 (1994) 32 41.

[2] Y Huang, R N Bird and O. Heidrich, A review of the use of recycled solid waste materials in asphalt pavements, Resour Conserv Recycl, 52, (2007) 58 73.

[3] S. D. Ramaswamy, E. W. Low, The effects of amino antistrip additives on stripping of bituminous mixes, High, Transp, 37(1990) 9 13.

[4] U Isacsson, and X Lu, Testing and appraisal of polymer modified road bitumens - state of the art, Mater. Struct. 28 (1995) 139 159.

[5] M Garcia-Morales, P Partal, F J Navarro, F Martinez Boza, C Gallegos, N González and M E Muñoz, Viscous properties and microstructure of recycled eva modified bitumen, Fuel, 83 (2004) 31 38.

[6] N Akmal and A. M Usmani, Asphalt, number one thermoplastic polymer, Polym News, 24 (2001) 136 40.

[7] A Ait Kadi, B Brahimi and M Bousmina, Polymer blends for enhanced asphalt binders, Polym Eng Sci, 36(2001) 1724 1733.

[8] F J Navarro, P Partal, F Martýinez-Boza, C Valencia and C Gallegos, Rheological characteristics of ground tire rubber modified bitumens, Chem Eng J, 89(2002)53 61. 
[9] S Shuler and D Hanson, Improving durability of open permits unrestricted use, provided the original author and graded friction courses, Transport Res Rec, 1259 source are credited.

(1990) 35 41. (book)

[10] 10.A Aksoy, K Samlioglu, S Tayfur and H Özen, Effects of various additives on the moisture damage sensitivity of asphalt mixtures, constr. Build. Mater, 19(2005) 11 18.

[11]C Gorkem and B Sengoz, Predicting stripping and moisture induced damage of asphalt concrete prepared with polymer modified bitumen and hydrated lime, constr. Build. Mater, 23(2008)2227 2236.

[12]C Giavarini and G Rinaldi, Development of new adhesion agents for asphalt cement, Ind. Eng. Chem. Res, 28(1989) 1231 1236.

[13]R Prigmore, S Whitehouse and A Bowe, Treatment of waste plastic material, WIPO Patent No. 2009118546 , Geneva, Switzerland: World Intellectual Property Organization, 2009.

[14]T Yupeng, Waste plastics environment friendly treatment method, Chinese Patent CN101733862 (A1), 2010.

[15] S Hirose, H Yasuda and H Takano, Treatment method of waste plastics, Japanese Patent JP2007244957 (A1), 2007.

[16]Christiane Raab, Manfred N Partl and A.E.H.O.A.E. Halim, Evaluation of Interlayer Shear Bond Devices for Asphalt Pavements, Balt J Road Bridge E, 4 (2009) 186 195

[17]H.L Robinson, Polymers in asphalt, Smithers Rapra Technology, United Kingdom, 2004.

[18] R Vasudevan, S K Nigam, R Velkennedy, A R C Sekar and B Sundarakannan, Utilization of waste polymers for flexible pavement and easy disposal of waste polymers, Proceedings of the International Conference on Sustainable Solid Waste Management, (2007)5 7.

[19]J Read and D Whiteoak, R. Hunter, Robert Hunter, The shell bitumen handbook, Thomas Telford Services Limited, (2003).

[20]S Hinislioglu and E Agar, Use of waste high density polyethylene as bitumen modifier in asphalt concrete mix, Mater. Lett, 58(2004)267 271.

[21] Q Lu and $\mathrm{J} \mathrm{T}$ Harvey, Long-term effectiveness of antistripping additives: Laboratory evaluation, Transportation research record: J. Transport. Res. Board, 1(1970) 14 24.

\section{About The License}

(C) 2019 The Authors. This work is licensed under a Creative Commons Attribution 4.0 International License which 